\title{
One Year Follow-up Result of Doppler-guided Hemorrhoidal Artery Ligation and Recto-Anal Repair in 97 Consecutive Patients
}

\author{
Wan Jo Jeong, Sung Wook Cho, Kyung Tae Noh $^{1}$, Soon Sup Chung ${ }^{1}$ \\ Department of Surgery, Seoul Red Cross Hospital, Seoul; ${ }^{1}$ Department of Surgery, Ewha Womans University School of Medicine, Seoul, Korea
}

Purpose: Doppler-guided hemorrhoidal artery ligation and recto-anal repair (DG-HAL \& RAR) is known for low recurrence, high patient satisfaction, and less postoperative pain. The purpose of this study is to analyze the 1-year follow-up results in patients who underwent a DG-HAL \& RAR and to establish the benefits of the procedure.

Methods: Among the hemorrhoid patients who were admitted to our hospital from March 2008 to May 2010 and who underwent a DG-HAL \& RAR, 97 patients who were followed up for a year were investigated. Recurrence, complications, admission period, difference in preoperative and postoperative pain, operation time, and time to return to daily activities were investigated.

Results: The average admission period was $1.6 \pm 1.1$ days. Pain at postoperative day 7 showed no significant difference from preoperative pain $(\mathrm{P}>0.05)$. The operation time was $34.0 \pm 7.3$ minutes on average, and return to daily activities was timed at $2.3 \pm 2.0$ days postoperatively. At the one year follow-up, no serious complications were noted, and preoperative symptoms recurred only in 14 patients (14.4\%).

Conclusion: In most patients with hemorrhoids, excluding those with severe prolapsed hemorrhoids, less pain, no serious complications, and good long-term outcome can be expected from a DG-HAL \& RAR.

Keywords: Hemorrhoids; Doppler; Ligation; Recto-anal repair

\section{INTRODUCTION}

The hemorrhoid is the most common anal disease. Depending on its symptoms, non-invasive treatments such as band ligation, cryotherapy, and sclerotherapy and invasive treatments such as a hemorrhoidectomy have been used to treat hemorrhoids. Among the aforementioned treatments, the hemorrhoidectomy has been most commonly used, but has disadvantages of severe postoperative pain, longer time to return to daily living, and complications

Received: July 25, 2011 Accepted: September 4, 2011

Correspondence to: Soon Sup Chung, M.D.

Department of Surgery, Ewha Womans University School of Medicine,

911-1 Mok 5-dong, Yangcheon-gu, Seoul 158-710, Korea

Tel: +82-2-2650-2517, Fax: +82-2-2765-5681

E-mail:gs3945@gmail.com

(c) 2011 The Korean Society of Coloproctology

This is an open-access article distributed under the terms of the Creative Commons Attribution NonCommercial License (http://creativecommons.org/licenses/by-nc/3.0) which permits unrestricted noncommercial use, distribution, and reproduction in any medium, provided the original work is properly cited. such as anal stricture [1]. To overcome these disadvantages, new treatment methods such as the procedure for prolapsed hemorrhoid (PPH) or Doppler-guided hemorrhoidal artery ligation have been introduced [2]. Doppler-guided hemorrhoidal artery ligation has been reported to have a low recurrence rate of less than $10 \%$, $90 \%$ or higher patient satisfaction, and minimal pain [3]. It achieves good outcomes in a relatively short time in the treatment of early hemorrhoid where bleeding or anal discomfort are main symptoms. However, it has a limitation in the treatment of advanced hemorrhoids where anal prolapse is the main symptom. For this reason, the simultaneous conduct of artery ligation and recto-anal repair has been recently introduced and has been reported to achieve good treatment outcomes [4]. In this research, a one-year followup study was conducted on hemorrhoidal patients who had undergone Doppler-guided Hemorrhoidal artery ligation and rectoanal repair (DG-HAL \& RAR) to investigate its outcomes and effectiveness. 


\section{METHODS}

This was a prospective cohort study conducted on 97 patients who underwent a one-year follow-up study from among patients who had been diagnosed with hemorrhoids and then had undergone a DG-HAL \& RAR in the authors' hospital from March 2008 to May 2010. Examinations of the surgery outcomes through outpatient visits were conducted three times: 7 days, one month, and three months after discharge. One year after discharge, recurrence and complications were followed-up via a telephone interview or outpatient visit. Recurrence was considered to have occurred in cases of re-bleeding or re-prolapse. Pre- and postoperative pain, operation time, and time to return to ordinary living were also examined. The comparison of preoperative and postoperative pain was assessed using a visual analog scale of $0-10$ ( 0 , no pain; 10 , the worst pain imaginable).

The surgery was conducted on internal hemorrhoidal patients with Goligher grade 2-4 hemorrhoids. Patients who were diagnosed with concomitant anal diseases such as anal fissure or fistula or with an incarcerated hemorrhoid or thrombotic hemorrhoid on preoperative anal examination were excluded from the surgery. All patients underwent the surgery in the lithotomy position. An anoscope equipped with a Doppler probe was inserted into the anus, together with a specially prepared sleeve. Then, the Doppler probe was placed around $3-5 \mathrm{~cm}$ from the upper dentate line to identify the branch of the upper rectal artery. The accurate detection of the branch of the upper rectal artery was confirmed via Doppler sound and the vessel depth displayed on the apparatus screen. Subsequently, a figure of eight suture was conducted using vicryl 2-0 at the rectal mucosa where the artery had been identified using the anoscope. After accurate ligation had been confirmed via loss of the Doppler vein signal, it was firmly ligated using a pusher. Next, after the hemorrhoidal mass had been exposed between the anoscope and the sleeve by rotating the anoscope, recto-anal repair was conducted in a way that hemorrhoidal mass was sequentially sutured from the artery ligation site to $5 \mathrm{~mm}$ of the upper dentate line by using the vicryl used for artery ligation and was firmly ligated in place on the rectal mucosa. The aforementioned procedure was performed on 6 sites (toward 1, 3, 5, 7, 9, and 11 o'clock) starting from the lower middle of the anus and going clockwise. The surgery was completed when no more prolapsed hemorrhoid or artery signal was found.

The data of this study are expressed as mean \pm SD. A statistical analysis was conducted using SPSS ver. 12.0 (SPSS Inc., Chicago, IL, USA). The difference between pre-operative and post-operative pain was tested via the Wilcoxon signed rank test. $\mathrm{P}<0.05$ was considered statistically significant.

\section{RESULTS}

The patients' mean age was $51.7 \pm 13.2$ years (range, 22 to 87 years), and the patients consisted of 62 males and 35 females. The most common main symptom observed in the patients before surgery was prolapsed lesion (83 cases), and anal bleeding ( 55 cases), defecation pain (22 cases), constipation (12 cases), and fecal incontinence ( 2 cases) were also observed. Both anal prolapsed lesions and bleeding were observed in 35 patients (Table 1). The preoperative disease stages included grade 2 hemorrhoids in 13 patients (13.4\%), grade 3 hemorrhoids in 68 patients (70.1\%), and grade 4 hemorrhoids in 16 patients (16.5\%) according to Goligher classification. Five patients had previous hemorrhoid surgery at least once. Most patients had had hemorrhoidal symptoms for more than one year, and some patients had had them for more than 30 years.

Anesthesia included systemic anesthesia, with intravenous anesthesia in 82 patients (84.5\%), spinal anesthesia in 9 patients (9.2\%), and local anesthesia in 6 patients (6.3\%). In cases of patients being afraid of systemic anesthesia or underlying diseases such as clotting disorder, spinal anesthesia or local anesthesia was conducted instead of systemic anesthesia. The patients were hospitalized on the day of surgery or one day before surgery, and underwent the surgery. The mean hospitalization period was $1.6 \pm 1.1$ days. Most patients were discharged on the day of surgery. However, 16 patients were discharged one day after the surgery, and four patients and one patient were discharged two days after the surgery and five days after the surgery, respectively. The mean operation time was $34.0 \pm 7.3$ minutes. The number of blood vessels to which ligation was conducted was mean $5.9 \pm 0.5$. Recto-anal repair was conducted an average of $5.8 \pm 0.5$ times.

In a survey on the return to daily living, patients answered that

Table 1. Demographic characteristics of hemorrhoid patients

\begin{tabular}{lc}
\hline Characteristics & Values \\
\hline Age (yr) & $51.7 \pm 13.2$ \\
Sex (M:F) & $62: 35$ \\
Chief complaints & \\
Protrusion & 83 \\
Anal bleeding & 55 \\
Anal pain & 22 \\
Constipation & 12 \\
Anal incontinence & 2 \\
Goligher grade & \\
॥ & $13(13.4)$ \\
III & $68(70.1)$ \\
IV & $16(16.5)$ \\
Anesthesia & \\
General anesthesia & $82(84.5)$ \\
Spinal anesthesia & $9(9.2)$ \\
Local anesthesia & $6(6.3)$ \\
\hline
\end{tabular}

Values are presented as number (\%). 
Table 2. Changes of anal pain

\begin{tabular}{lccc}
\hline & Preop pain & $\begin{array}{c}\text { Postop pain } \\
(2 \mathrm{hr})\end{array}$ & $\begin{array}{c}\text { Postop pain } \\
(>7 \text { day })\end{array}$ \\
\hline Visual analog scale & $1.0 \pm 2.2$ & $2.4 \pm 3.0^{\mathrm{a}}$ & $0.8 \pm 1.4^{\mathrm{b}}$ \\
\hline
\end{tabular}

${ }^{2}$ Difference between preoperative pain and postoperative pain $(2 \mathrm{hr}), \mathrm{P}<0.05$.

${ }^{b}$ Difference between preoperative pain and postoperative pain (7 day), $\mathrm{P}>0.05$.

they were able to return to their daily lives, on average, $2.3 \pm 2.0$ days after the surgery. The pain score was shown to increase from $1.0 \pm 2.2$ points before the surgery to $2.4 \pm 3.0$ points two hours after the surgery to $0.8 \pm 1.4$ points 7 days after the surgery. No significant difference in pain score was found 7 days after the surgery (Table 2). The result of the follow-up conducted 7 days after the surgery showed tenesmus in 19 patients, bleeding in 8 patients, voiding dysfunction in 7 patients, and prolapse in 2 patients. However, no serious complications requiring re-hospitalization were observed. In a follow-up study conducted one month after the surgery, tenesmus, bleeding, and prolapse were observed in 6,4, and 5 patients, respectively. When the four patients with bleeding were examined using an anoscope, two patients showed bleeding caused by acute anal fissure irrelevant to hemorrhoidal bleeding. In the outpatient visit three months after the surgery, no patient complained of tenesmus, and anal prolapse and bleeding were observed in 5 and 2 patients, respectively. Two patients with acute anal fissure showed no further bleeding after they underwent conservative treatments. In a follow-up study conducted one year after the surgery, the preoperative symptoms recurred in 14 patients (14.4\%). Four patients complained of defecation bleeding, of whom two patients had grade 2 internal hemorrhoids and two patients had grade 3 internal hemorrhoids. Ten patients complained of prolapse, of whom four patients had grade 3 internal hemorrhoids and 6 patients had grade 4 internal hemorrhoids. The score of surgery satisfaction one year after the surgery was $7.4 \pm 2.9$ points, and 76 patients $(78.3 \%)$ replied in a survey that they would recommend the surgery to other people.

\section{DISCUSSION}

The hemorrhoid is the most common disease among anal diseases and is known to be caused by anal cushion descent or abnormal congestion of the internal hemorrhoidal venous plexus. Various treatment methods have been developed and used to date [2]. Among the aforementioned methods, the hemorrhoidectomy is the most fundamental treatment method and has been used for a long time. It is a good method to eliminate the pathophysiological factors of hemorrhoids, but has disadvantages of having an approximately $10 \%$ postoperative complication rate [2], requiring time for treatment, and requiring significant time to return to daily living. To overcome these disadvantages, new operation methods have been developed to minimize postoperative pain and to return to daily living as soon as possible. The PPH, which was first intro- duced by Longo et al. in 1993, is a surgery method that effectively blocks the blood flow of the upper rectal artery into the hemorrhoid by segmentally resecting the rectal mucosa with a regular size in a circular form and suturing them automatically and that fixes the descended rectal mucosa by uplifting it. It has advantages of less postoperative pain, shorter hospitalization period, and simpler procedure. However, it has a disadvantage in that it may cause serious complications such as bleeding, perforation, peritonitis, and fecal incontinence unless the resection plane is precisely sutured [57]. In addition, a long-term follow-up study reported that recurrence rate was higher for the PPH than for a hemorrhoidectomy [8].

Doppler-guided hemorrhoidal artery ligation, which was first introduced by Morinaga et al. [9] in 1995, is a surgery method that ligates and contracts hemorrhoid mass after detecting the upper rectal artery branch connected to each hemorrhoid through a Doppler signal, and compared to a conventional hemorrhoidectomy, no difference in the recurrence of preoperative symptoms was reported to have been found in a one-year follow-up study. A 3-year follow-up study including grade 4 hemorrhoids reported good outcomes, such as a $12 \%$ recurrence rate and fewer complications [10, 11]. However, artery ligation alone has limitations in the removal of the remaining anal mucosa and the treatment of a severely prolapsed hemorrhoid. Accordingly, the early apparatus was recently modified to simultaneously conduct artery ligation and RAR. Thus, it had an effect of fixing the upper anal canal upward after $\mathrm{PPH}$ suturing.

The conventional hemorrhoidectomy was conducted in the jackknife position in most cases whereas the technique used in this study was conducted in the lithotomy position. In the jack-knife position, the Doppler probe handle cannot be freely rotated in the leg-assembled position, which limits its use. However, in the lithotomy position, the Doppler probe can be freely rotated. Therefore, surgeons prefer the lithotomy position to the jack-knife position.

In this study, artery ligation and recto-anal repair each were conducted an average of mean six times. The mean operation time was approximately 35 minutes. No significant differences in the operation times and the numbers of artery ligations and recto-anal repairs were found among the patients. Meanwhile, in other studies $[4,12-16]$, the number of artery ligations was, on average, 5-10; particularly, the number of recto-anal repairs was a minimum of 1 and a maximum of 5 , which was less than the number recto-anal repairs conducted in this study. The reason for this difference is attributed to differences in the surgery methods. In this study, during artery ligation, the recto-anal repair was simultaneously conducted in such a manner that the prolapsed hemorrhoid mass on the Doppler probe's groove was sequentially sutured. Meanwhile, in other studies, each artery ligation was conducted for the entire anal canal in advance, and several prolapsed hemorrhoids were then separated, followed by recto-anal repair.

In a follow-up study conducted 7 days after the surgery, approximately $20 \%$ of the patients suffered from tenesmus, which was higher than the frequency reported in another study [12]. This is 
likely attributable to the fact that the number of recto-anal repairs was higher in this study than in the other study. A further comparative study is required to obtain more evidence.

In a follow-up study conducted one year after the surgery, recurrence, including re-bleeding, was observed in 14 patients (14.4\%), which was a higher recurrence rate than that shown in other studies $[4,12-16]$. The recurrence rates were $15 \%, 9 \%$, and $38 \%$ for grade 2 , grade 3 , and grade 4 hemorrhoids, respectively. When only prolapse was considered, recurrence occurred in $10 \%$ of the patients. In this case, no significant difference in recurrence rate was found between this study and other studies. However, the direct comparison is not really reliable as each study had different recurrence criteria. Only prolapse was defined as recurrence in one study [16] whereas prolapse or bleeding was defined as recurrence in this study. In addition, symptoms requiring surgery were defined as recurrence in another study [15]. Thus, an accurate comparison may be difficult. Faucheron et al. [16] conducted a follow-up study of approximately 34 months after a study conducted on 100 patients with grade 4 hemorrhoids and reported a $9 \%$ recurrence rate. This rate is significantly lower than the $38 \%$ recurrence rate for grade 4 hemorrhoids of this study. This difference is likely attributable to the different criteria of grade 4 hemorrhoid. In this study, prolapsed hemorrhoids that were prolapsed over the entire anus were mainly included in the criteria of grade 4 hemorrhoid, but in the study conducted by Faucheron et al. [16], partially prolapsed hemorrhoids were mainly included. A study conducted by Szmulowicz et al. [17] also reported that $65 \%$ of recurrent patients had large-sized hemorrhoids prolapsed over the entire anus. A follow-up study conducted for more than one year showed that DG-HAL \& RAR was a safe and effective treatment method for most hemorrhoids except grade 4 hemorrhoids with severe prolapse.

This study has a limitation in that it was conducted with a restricted number of surgeons in a single institution. However, as this technique is currently being used in other medical institutions in addition to the authors' hospital, a long-term, large-scale multicenter study could be conducted to compare this technique with other surgery methods in the future.

A DG-HAL \& RAR for the treatment of most hemorrhoids, except hemorrhoid with severe prolapsed, achieved a satisfactory outcome without significant pain and complications. A further longterm, large-scale multicenter follow-up study is required. Furthermore, a study on the treatment of hemorrhoids associated with other anal diseases is required.

\section{CONFLICT OF INTEREST}

No potential conflict of interest relevant to this article was reported.

\section{REFERENCES}

1. Chen JS, You JF. Current status of surgical treatment for hemorrhoids: systematic review and meta-analysis. Chang Gung Med J
2010;33:488-500.

2. Hardy A, Chan CL, Cohen CR. The surgical management of haemorrhoids: a review. Dig Surg 2005;22:26-33.

3. Giordano P, Overton J, Madeddu F, Zaman S, Gravante G. Transanal hemorrhoidal dearterialization: a systematic review. Dis Colon Rectum 2009;52:1665-71.

4. Satzinger U, Feil W, Glaser K. Recto anal repair (RAR): a viable new treatment option for high-grade hemorrhoids. One year results of a prospective study. Pelviperineology 2009;28:37-42.

5. Molloy RG, Kingsmore D. Life threatening pelvic sepsis after stapled haemorrhoidectomy. Lancet 2000;355:810.

6. Cheetham MJ, Mortensen NJ, Nystrom PO, Kamm MA, Phillips RK. Persistent pain and faecal urgency after stapled haemorrhoidectomy. Lancet 2000;356:730-3.

7. Herold A, Kirsch JJ. Pain after stapled haemorrhoidectomy. Lancet 2000;356:2187.

8. Jayaraman S, Colquhoun PH, Malthaner RA. Stapled hemorrhoidopexy is associated with a higher long-term recurrence rate of internal hemorrhoids compared with conventional excisional hemorrhoid surgery. Dis Colon Rectum 2007;50:1297-305.

9. Morinaga K, Hasuda K, Ikeda T. A novel therapy for internal hemorrhoids: ligation of the hemorrhoidal artery with a newly devised instrument (Moricorn) in conjunction with a Doppler flowmeter. Am J Gastroenterol 1995;90:610-3.

10. Bursics A, Morvay K, Kupcsulik P, Flautner L. Comparison of early and 1-year follow-up results of conventional hemorrhoidectomy and hemorrhoid artery ligation: a randomized study. Int J Colorectal Dis 2004;19:176-80.

11. Faucheron JL, Gangner Y. Doppler-guided hemorrhoidal artery ligation for the treatment of symptomatic hemorrhoids: early and three-year follow-up results in 100 consecutive patients. Dis Colon Rectum 2008;51:945-9.

12. Theodoropoulos GE, Sevrisarianos N, Papaconstantinou J, Panoussopoulos SG, Dardamanis D, Stamopoulos P, et al. Dopplerguided haemorrhoidal artery ligation, rectoanal repair, sutured haemorrhoidopexy and minimal mucocutaneous excision for grades III-IV haemorrhoids: a multicenter prospective study of safety and efficacy. Colorectal Dis 2010;12:125-34.

13. Walega P, Krokowicz P, Romaniszyn M, Kenig J, Sałowka J, Nowakowski $\mathrm{M}$, et al. Doppler guided haemorrhoidal arterial ligation with recto-anal-repair (RAR) for the treatment of advanced haemorrhoidal disease. Colorectal Dis 2010;12:e326-9.

14. Infantino A, Bellomo R, Dal Monte PP, Salafia C, Tagariello C, Tonizzo CA, et al. Transanal haemorrhoidal artery echodoppler ligation and anopexy (THD) is effective for II and III degree haemorrhoids: a prospective multicentric study. Colorectal Dis 2010;12: 804-9.

15. Ratto C, Donisi L, Parello A, Litta F, Doglietto GB. Evaluation of transanal hemorrhoidal dearterialization as a minimally invasive therapeutic approach to hemorrhoids. Dis Colon Rectum 2010;53: 803-11.

16. Faucheron JL, Poncet G, Voirin D, Badic B, Gangner Y. Doppler- 


\section{Coloproctology wan Jo Jeong, et al.}

guided hemorrhoidal artery ligation and rectoanal repair (HALRAR) for the treatment of grade IV hemorrhoids: long-term results in 100 consecutive patients. Dis Colon Rectum 2011;54:226-31.
17. Szmulowicz UM, Gurland B, Garofalo T, Zutshi M. Doppler-guided hemorrhoidal artery ligation: the experience of a single institution. J Gastrointest Surg 2011;15:803-8. 